\title{
Opinión del Comité Consultivo de Inmunizaciones de la Sociedad Chilena de Infectología en relación a los brotes de hepatitis A en Chile
}

\author{
Rodolfo Villena, Jan Wilhelm, Ximena Calvo, Jaime Cerda, Carola Escobar, \\ Gabriela Moreno, Liliana Veliz y Marcela Potin
}

\section{Statement of the Advisory Committee on Immunizations of Sociedad Chilena de Infectología about outbreaks of hepatitis A in Chile}

This document represents the position of the Chilean Infectious Diseases Society Advisory Committee on Immunization Practices regarding hepatitis A epidemiological situation in Chile. The recommendations are based on local epidemiological data, the hepatitis A virus infection characteristics and the global experience with the available vaccines. In relation to hepatitis A, Chile is no longer a highly endemic area but actually an intermediate one, currently concentrating cases in individuals over 15 years of age, with frequent outbreaks. In 2017 we have seen an important outbreak of genotype 1A in men who have sex with men (MSM). The current endemic, the presence of regular outbreaks, the frequency of natural disasters in Chile, together with the availability of safe, effective vaccines and local cost-effectiveness studies led us to propose measures for outbreak control for high risk groups protection and mid and long term, including a more definitive solution which is universal vaccination against this disease in small children.

Palabras clave: Brotes; hepatitis A; control; vacunas.

Key words: Outbreaks; hepatitis A; control; vaccines
Conflictos de interés: Rodolfo Villena declara haber trabajado como asesor científico de vacunas para Novartis entre 2012 y 2015 , y como expositor en simposios de vacunas auspiciados por Pfizer, Sanofi Pasteur, GSK y MSD. EI resto de los autores no declaran conflicto de interés.

Recibido: 5 de junio de 2017

Correspondencia a: Jan Paul Wilhelm Janpaulwilhelm@gmail.com

\section{Situación epidemiológica actual}

$\mathrm{E}$ n Chile, la hepatitis A se presenta en forma de endemia intermedia, con ciclos epidémicos que desde 1990 ocurren cada 3 o 5 años, y tasas de incidencia que han variado desde 70,9 hasta 3,2 por 100 mil habts., entre los años 2002 y 2010 . A partir del año 2005, 70\% de los casos se han presentado en sujetos mayores de 15 años.

La distribución de los casos ha sido heterogénea a lo largo del país, encontrándose las tasas más altas en las regiones de Tarapacá, Arica y Parinacota, que llegan a concentrar $45 \%$ de los casos del país, con tasas de 173 por 100 mil habts. en el año 2003, a pesar de que su población sólo constituye $3 \%$ de la población nacional. La investigación epidemiológica mostró que el factor de riesgo más importante era el consumo de alimentos expendidos en la vía pública.

Por este motivo, se implementó un programa de vacunación contra hepatitis A con dosis única, en niños entre 18 meses y 6 años de edad, logrando bajar la tasa a 8,0 por 100 mil habts.

En los años 2015 y 2016, la Región del Bío Bío presentó la tasa más alta del país, con valores de 8 y 24,4 por 100 mil habts. respectivamente, encontrándose entre las posibles causas las consecuencias del terremoto del 2010, por lo que igualmente se implementó una estrategia de vacunación con monodosis de vacuna de hepatitis A.

A la semana epidemiológica (SE) 18 del año 2017 se han presentado 706 casos en el pais, con una tasa acumulada de 3,8 por 100 mil habts., lo que implica un aumento de 53\% respecto del mismo período del año 2016, concentrándose $78 \%$ de los casos en el grupo etario entre 15 y 39 años, con una relación varones mujeres de 4:1. En todas las semanas del año 2017, los casos de hepatitis A han estado sobre lo esperado. Las regiones que presentan aumento de sus tasas respecto del mismo período de 2016 son, en orden geográfico norte a sur, Antofagasta, Valparaíso, Región Metropolitana (RM), O’Higgins y Maule.

Este aumento de casos ha predominado en la RM, donde se ha detectado un brote desde la SE 47 de 2016. A la semana 14 de este año, se han notificado 253 casos con una tasa de 3,4 por 100 mil habts., lo que implica un aumento de más de seis veces comparado con el año 2016, en que a la misma fecha se habían notificado 40 casos, con una tasa de 0,5 por 100 mil habts. La investigación epidemiológica muestra que no hay aumento en el grupo bajo 15 años de edad, y que $85 \%$ de los casos ocurre en varones entre 15 y 39 años.

Los hombres han tenido casi siete veces más riesgo 
de enfermar que las mujeres, mientras que, en el grupo de mayor riesgo, el de 25 a 29 años, esta razón ha sido de casi 17 veces. No se han identificados factores de riesgo asociados al consumo de alimentos o hábitos de higiene; sin embargo, la exposición oro-anal en hombres que tienen sexo con hombres (HSH) sería uno de los principales factores de riesgo. Los casos se concentran principalmente en las comunas de Santiago Centro, Providencia y Ñuñoa.

El análisis filogenético de los virus circulantes en el brote de la RM, indica que corresponden al genotipo 1A, el que concuerda con el descrito en los brotes de hepatitis A en HSH reportados a principios de 2017 en Alemania, Países Bajos y otros países europeos y que no había sido identificado en muestras analizadas en períodos anteriores en nuestro país. El SEREMI de la RM ha intensificado la vigilancia epidemiológica y amplió las medidas de bloqueo de contactos de casos hasta los 40 años con vacuna anti hepatitis $A$.

En la Región de Antofagasta se han registrado 41 casos de hepatitis A a la SE 18, la segunda mayor tasa en el país, con 6,4 casos por 100.000 habts., más de seis veces el valor de la mediana 2009-2015 (n: 7). El mayor riesgo se presenta en el grupo de 25 a 29 años, seguido del grupo de 20 a 24 años con tasas de 20,7 y 16,0 casos por 100.000 habts., respectivamente. El grupo de 5 a 9 años también presenta una tasa elevada respecto al resto de los grupos con 12,2 casos por 100.000 habts. Los hombres en esta región presentan casi dos veces más riesgo que las mujeres. Dentro de los factores de riesgo se constató que $51 \%$ de los pacientes declaró haber consumido alimentos en la vía pública y otro $51 \%$ mencionó vectores (moscas, cucarachas y roedores) en los domicilios. El 94\% de los casos corresponden a personas de nacionalidad chilena. En este caso, el virus circulante también pertenece al genotipo $1 \mathrm{~A}$, pero no está relacionado con las cepas identificadas en el brote actual de la RM.

\section{Antecedentes}

El virus de la hepatitis A (VHA) pertenece a la familia Picornaviridae, posee un serotipo y cuatro genotipos. Su incubación puede ir entre 15 y 50 días y es altamente resistente a las condiciones ambientales. Es de reservorio humano exclusivo (personas asintomáticas o enfermos clínicos) y se transmite por vía fecal-oral (ciclo corto) o por medio de alimentos y aguas contaminadas (ciclo largo). Su epidemiología se relaciona estrechamente con condiciones higiénico-sanitarias y con el hecho de que la enfermedad es más manifiesta y complicada a mayor edad de infección. La mayoría de los casos en niños bajo 5 años de edad son asintomáticos, aunque pueden excretar el virus por semanas. A los 5 años de edad, sólo
$30 \%$ de los casos son sintomáticos, mientras que $70 \%$ de los casos en adultos que ingieren el virus presentarán manifestaciones clínicas, siendo las complicaciones y hospitalizaciones más frecuentes en este grupo etario. En Latinoamérica es la causa más frecuente de trasplante de hígado, debido a su complicación más grave, la falla hepática fulminante. Las mejoras sanitarias y de educación en Chile nos han permitido transitar desde una endemia alta a una intermedia, lo cual nos ubica en un escenario de especial riesgo, pues desplaza los casos sintomáticos hacia la adultez.

\section{Las vacunas}

Las vacunas contra hepatitis A actualmente disponibles en nuestro país son vacunas inactivadas, muy eficaces y seguras. Están registradas en Chile para su uso en personas a partir de un año de edad, son intercambiables entre ellas y pueden ser co-administradas con otras vacunas. $\mathrm{Su}$ inmunogenicidad ha sido ampliamente demostrada en ensayos clínicos y una sola dosis de la vacuna induce células de memoria en forma semejante a la infección natural por VHA.

La Organización Mundial de la Salud recomienda la incorporación de la vacuna contra la hepatitis A en los Programas Nacionales de Inmunización (PNI) desde los 12 meses de vida en los países con transición epidemiológica, de endemia alta a intermedia, considerando estudios de costo efectividad, ya sea en esquemas de dos dosis o monodosis (excepto en grupos de riesgo, en quienes debe observarse el uso de dos dosis). Un estudio local de costo-beneficio efectuado en el año 2004, mostró que si se incorporara esta vacunación en un esquema de dos dosis ( 18 y 24 meses o 18 y 54 meses), se evitarían más de $86 \%$ de los casos en los grupos etarios respectivos y sus contactos y, además, se evitarían al menos 70 infecciones fatales, el Estado recuperaría su inversión total en 20 años y se ahorrarían más de 4.600 años de vida ajustados por calidad. Quezada y cols., en el 2008, sugirieron que, vacunando rutinariamente con dos dosis, a los 12 y 18 meses de edad, se reducirían sustancialmente los casos de hepatitis A, siendo menos costoso que la no vacunación, desde el sexto año de implementación.

Argentina incorporó rutinariamente la vacunación contra hepatitis A en dosis única a los 12 meses de vida desde el año 2005, demostrando ser una estrategia efectiva, inductora de inmunidad comunitaria y persistiendo la inmunogenicidad hasta al menos nueve años posteriores a la vacunación.

La experiencia de epidemias de hepatitis A, cada tres a cinco años, la recurrencia de desastres naturales en nuestro país, y la mantención de la transmisión por ciclos largos en situaciones de endemia intermedia, hacen prever que el 
problema continuará en el tiempo de no mediar intervenciones más globales. El uso de medidas de control de brote o la vacunación de grupos específicos no ha demostrado ser efectivo en la eliminación de la circulación del virus de manera persistente. El control y eliminación del VHA en Chile es posible, dado que el reservorio del VHA es exclusivamente humano; no se ha descrito infección ni excreción crónicas; existe sólo un serotipo, de distribución universal; el diagnóstico es confiable y está estandarizado y disponemos de vacunas eficaces y seguras, con estudios de costo-efectividad locales.

\section{Recomendaciones del Comité Consultivo de Inmunizaciones de la Sociedad Chilena de Infectología:}

\section{Medidas a mediano y largo plazo}

- Extender progresivamente la vacunación programática anti-hepatitis A, incorporándola a nuestro PNI en dosis única en niños a los 18 meses de vida, priorizando en el tiempo a las regiones con mayor número de casos, como actualmente sucede en la RM.

\section{Medidas focalizadas en grupos de riesgo}

- Vacunar en forma regular a HSH y personas con infección por VIH y sus contactos.

\section{Medidas de control de brote}

- Realizar una campaña de vacunación contra el VHA en la población menor de 40 años de sexo masculino en las regiones con mayor número de casos, que en el momento actual corresponden a RM, Antofagasta y Bío-Bío. Además, continuar la aplicación de acciones de bloqueo vacunando a los contactos de los casos.

\section{Medidas adicionales}

- Mantener campañas educativas en medidas de prevención de la infección por VHA.

- Reforzar las alertas epidemiológicas de vigilancia de hepatitis A.

- Mejorar la disponibilidad de exámenes de laboratorio para confirmar la etiología de los brotes de hepatitis.

Estas recomendaciones están en la línea de las entregadas el año 2003 por la Sociedad Chilena de Infectología en relación al control de la hepatitis A en nuestro país.

\section{Resumen}

El presente documento corresponde a la opinión del Comité Consultivo de Inmunizaciones de la Sociedad Chilena de Infectología, en relación a los brotes de hepatitis A en Chile. Las recomendaciones emitidas se basan en antecedentes epidemiológicos, características de la infección por virus hepatitis A(VHA) y en la experiencia mundial con las vacunas disponibles En relación a la infección por virus hepatitis A, Chile ha transitado de una endemia alta a una endemia intermedia, concentrándose actualmente los casos en personas mayores de 15 años, con brotes frequentes, uno de ellos este año causado por el genotipo 1A del VHA que se concentró en hombres que tienen sexo con hombres (HSH). La endemia actual, la presencia de brotes regulares, la frecuencia de desastres naturales en el país como terremotos e inundaciones sumado a la disponibilidad de vacunas eficaces, seguras y con estudios de costo efectividad locales, nos llevan a plantear medidas para el control de brotes, medidas focalizadas en grupos de riesgo y especialmente medidas a mediano y largo plazo, que incluyen la vacunación universal de niños contra esta enfermedad.

\section{Referencias bibliográficas}

1.- O’Ryan M, Valenzuela M T, Santolaya M E, Abarca K, Lagos R, Cofré J. Declaración: La Sociedad Chilena de Infectología frente a la situación epidemiológica de hepatitis A en Chile. Rev Chilena Infectol 2003; 20 (3): 199-201.

2.- Aggarwal R, Goel A. Hepatitis A: epidemiology in resource-poor countries. Curr Opin Infect Dis 2015; 28 (5): 488-96.

3.- Ibarra H. Cambios en la epidemiología de las hepatitis virales en Chile y consideraciones en estrategias de prevención. Rev Med Chile 2007; 135: 229-39.
4.- http://epi.minsal.cl/wp-content/uploads/ 2017/05/Informe_HepA_SE182017.pdf

5.- Valenzuela M T, Jacobs $\bar{R} \mathrm{~J}$, Arteaga $\mathrm{O}$, Navarrete M S, Meyerhoff A S, Innis BL Costeffectiveness of universal childhood hepatitis A vaccination in Chile. Vaccine 2005; 23 (32): 4110-9.

6.- Quezada A, Baron-Papillon F, Coudeville L, Maggi L. Universal vaccination of children against hepatitis A in Chile: a cost-effectiveness study. Rev Panam Salud Publica. 2008 May; 23 (5): 303-12.

7.- Weekly Epidemiological Record, WHO position paper on hepatitis A vaccines. No. 2829,13 july 2012.

8.- Vizzotti C, González J, Gentile A, Rearte A,
Ramonet M, Cañero-Velasco M C, et al. Impact of the single-dose immunization strategy against hepatitis A in Argentina. Pediatr Infect Dis J 2014; 33 (1): 84-8.

9.- Urueña A, González J E, Rearte A, Pérez Carrega M E, Calli R, Pagani M F, et al. Singledose universal hepatitis A immunization in oneyear-old children in Argentina: High prevalence of protective antibodies up to 9 years after vaccination. Pediatr Infect Dis J 2016; 35 (12): 1339-42.

10.- Vizzotti C, Pippo T, Urueña A, Altuna J, Palópoli G, Hernández M L, et al. Economic analysis of the single-dose immunization strategy against hepatitis A in Argentina. Vaccine. 2015; 33 Suppl 1: A227-32. 